\title{
Regulation of the invasion and metastasis of human glioma cells by polypeptide $\mathrm{N}$-acetylgalactosaminyltransferase 2
}

\author{
JUN LIU $^{1,2}$, LINGYAN YANG ${ }^{1}$, MEIFANG JIN ${ }^{1}$, LAN XU $^{1}$ and SHILIANG WU ${ }^{1}$ \\ Departments of ${ }^{1}$ Biochemistry and Molecular Biology, and ${ }^{2}$ Anatomy, \\ Medical College, Soochow University, Suzhou, Jiangsu 215123, P.R. China
}

Received June 29, 2011; Accepted August 17, 2011

DOI: $10.3892 / \mathrm{mmr} .2011 .569$

\begin{abstract}
Polypeptide, N-acetylgalactosaminyltransferase 2 (ppGalNAc-T2), is a member of the ppGalNAcT family which is the initial glycosyltransferase in O-glycan synthesis. Our previous studies have demonstrated that ppGalNAc-T2 plays a vital role in the process of tumor emergence and development. In this study, we aimed to determine whether ppGalNAc-T2 is correlated with the invasion and metastasis of human glioma cells. PpGalNAc-T2 sense vectors and interference vectors constructed in our laboratory were successfully transfected into SHG44 and U251 cells, which were observed through fluorescence microscopy, RT-PCR and Western blot analysis. The metastasis of U251 cells was determined using a wound healing assay, and ppGalNAc-T2 was found to inhibit cell migration. By RT-PCR, glioma cell invasion assay showed that the expression of MMP-2 was increased in ppGalNAcT2-knockdown cells, while the expression of MMP-9 and TGF- $\beta 1$ was decreased in ppGalNAc-T2-overexpressing cells at the mRNA level. Meanwhile, the expression of TIMP-2 at the mRNA level was contrary to MMP-2. In summary, our findings indicate that ppGalNAc-T2 plays a potential role in glioma cell migration and invasion, suggesting a new molecular therapeutic target for human malignant glioma treatment.
\end{abstract}

\section{Introduction}

Glycans are one of the four basic components of cells and may also be the most abundant and diverse of nature's biopolymers. Glycans participate in many key biological processes including cell adhesion, molecular trafficking and clearance, receptor activation, signal transduction and endocytosis. Recent studies have also shown that glycan alterations in malignant cells take on a variety of forms and mediate key pathophysi-

Correspondence to: Dr Shiliang Wu, Department of Biochemistry and Molecular Biology, Medical College, Soochow University, Suzhou, Jiangsu 215123, P.R. China

E-mail: shiliang_wu@126.com

Key words: glioma, ppGalNAc-T2, MMP-2, invasion and metastasis ological events during the various stages of tumor progression (1-4). O-glycans are attached to the hydroxyl groups in serine (Ser) and threonine (Thr) residues and include O-linked $\mathrm{N}$-acetyl-D-glucosamine (GlcNAc), mannose, fucose and $\mathrm{N}$-acetylgalactosamine (GalNAc). The latter type of glycosylation, referred to as mucin-type O-glycosylation, is referred to simply as $\mathrm{O}$-glycosylation (5). Cancer cells may contain membrane-bound mucin-like glycoproteins that have domains rich in O-glycans. The gene expression of these glycoproteins is cell-specific and is often altered in cancer $(6,7)$.

Glycosyltransferases, responsible for glycosylation, transfer a sugar residue from an activated nucleotide sugar donor, to specific acceptor molecules, forming glycosidic bonds. Research on glycan function has revealed that the glycosyltransferases are essential for the development and physiology of living organisms. Mucin-type linkages (GalNAc $\alpha 1-O-S e r / T h r)$ are initiated by a family of glycosyltransferases called the UDP-N-acetylgalactosamine: polypeptide $\mathrm{N}$-acetylgalactosaminyltransferases (ppGalNAcTs, EC 2.4.1.41), which is a crucial regulatory step. These enzymes transfer GalNAc from the sugar donor UDP-GalNAc to serine and threonine residues forming an $\alpha$ linkage (8). Alteration of glycosyltransferase activities are likely important in cancer, as suggested by the ability of certain glycosyltransferases to control the formation of cancer-associated glycans. There have been considerable insights into this field. Rajpert-De Meyts et al concluded that simple mucin-type O-glycans and their transferases were developmentally regulated in the human testis, with profound changes associated with neoplasia (9). Liu et al confirmed that down-regulation of $\beta 3 \mathrm{Gn}-\mathrm{T} 8$ gene expression inhibited the growth of human gastric cancer (10). PpGalNAc-T2 is a member of the ppGalNAc-T family. Results from our previous studies have demonstrated that ppGalNAc$\mathrm{T} 2$ plays a vital role in the process of tumor emergence and development (11). In this study, we focused on ppGalNAc-T2 and glioma and aimed to determine whether ppGalNAc-T2 is correlated with cell invasion and migration.

Gliomas are the most common primary brain tumors. Despite advances in surgery, radiation and chemotherapy, malignant gliomas are still highly lethal tumors. The prognosis for patients with malignant gliomas remains poor. The pathophysiological processes of angiogenesis and tumor cell invasion play pivotal roles in glioma development and growth already in the earliest phase. The invasive glioma cells are 
found to migrate along myelinated fiber tracts of white matter and along blood vessel basement membranes (12). Invasion of tumor cells into normal tissue is thought to be a multi-factorial process, consisting of cell interactions with extra-cellular matrix (ECM) and with adjacent cells. Tumor cell invasion requires four distinct steps: i) detachment of invading cells from the primary tumor mass, ii) adhesion to ECM, iii) degradation of ECM, and (iv) cell motility and contractility (13).

ECM is thought to be a type of barrier for tumor cell invasion, and its cleavage and removal are more or less necessary for tumor cell migration (14). The most common proteases that degrade ECM to create the space for invading tumor cells are matrix metalloproteinases (MMPs). MMPs degrade various cell adhesion molecules, thereby modulating cell-cell and cellECM interactions (15). The role of MMPs in glioma invasion and migration is well documented. For instance, differential roles have been suggested whereby MMP-9 contributes primarily to invasion along established blood vessels, while MMP-2 regulates both invasion and angiogenesis (16). Furthermore, increased MMP-2, along with increased MMP-9 and MMP-3, has also been observed in a genetically defined model of SPARC-induced glioma invasion (17). The multifunctional cytokine transforming growth factor $\beta 1$ (TGF- $\beta 1$ ) plays a dual role in the process of carcinogenesis by promoting tumor progression by enhancing migration, invasion and survival of tumor cells (18). Furthermore, TGF- $\beta 1$ was reported to induce secretion and activation of MMP-2 and MMP-9 in cancer cell lines $(19,20)$.

Thus, this study was undertaken to evaluate the role of ppGalNAc-T2 in glioma invasion and metastasis by creating stable transfectants and evaluating them for invasive and metastatic potential in vitro. Herein, we mainly investigated the change in MMP-2, MMP-9, tissue inhibitor of metalloproteinase-2 (TIMP-2) and TGF- $\beta 1$ expression at the mRNA level. We further investigated the metastasis of glioma cells in vitro using a wound healing assay. Our findings indicate that ppGalNAc-T2 plays a useful role in regulating glioma invasion and metastasis, which may be used as a novel approach for cancer therapy.

\section{Materials and methods}

Materials. The glioma cell lines U251 and SHG44 were obtained from the School of Pharmacy, Soochow University. RPMI-1640 complete culture medium was purchased from Gibco (USA). TRIzol reagent was purchased from Invitrogen (USA). Anti-human ppGalNAc-T2 polyclonal antibody was produced from rabbits in our laboratory. Anti- $\beta$-actin antibody was purchased from Santa Cruz Biotechnology. Other reagents were commercially available in China.

Cell culture. Human glioma U251 and SHG44 cells were cultured in RPMI-1640 containing 10\% fetal bovine serum (FBS) in a humidified atmosphere with $5 \% \mathrm{CO}_{2}$ at $37^{\circ} \mathrm{C}$.

Plasmid identification and transfection. The pcDNA3.1ppGalNAc-T2 (ppGalNAc-T2 sense vectors), pEGFP-C1-ppGalNAc-T2 (ppGalNAc-T2 sense vectors), pSilenCircle-ppGalNAc-T2Si (ppGalNAcT2 interference vector) and pSilenCircle-ppGalNAc-T2Scr (control vectors) plasmids were constructed in our laboratory. The pcDNA3.1ppGalNAc-T2 and pEGFP-C1-ppGalNAc-T2 plasmids were identified by digestion with restriction enzymes BamHI and EcoRI, while the pSilenCircle-ppGalNAc-T2Si was digested by StuI (MBI, Fermentas, Lithuania). Plasmid DNA was purified as described in the EndoFree plasmid purification handbook (Qiagen, Ltd., Crawley, UK). Spectrophotometric analysis revealed the $260 / 280 \mathrm{~nm}$ ratio to be $\geq 1.80$. The purity of DNA preparations was confirmed again on a $1 \% \mathrm{w} / \mathrm{v}$ agarose gel.

U251 and SHG44 cells were plated at a density of $2 \times 10^{5}$ cells per well in 6-well plates and incubated for $24 \mathrm{~h}$. The U251 cells were then transfected with pEGFP-C1 (empty vector), pEGFP-C1-ppGalNAc-T2, pSilenCircle-ppGalNAcT2Scr and pSilenCircle-ppGalNAc-T2Si, while the SHG44 cells were transfected with pcDNA3.1 (empty vector) and pcDNA3.1-ppGalNAc-T2 by Lipofectamine 2000 (Invitrogen), followed by selection with G418 $(500 \mu \mathrm{g} / \mathrm{ml})$. Individual clones were selected and analyzed. The stable cells were correspondingly named PE vs. PE-T2, Scr-T2 vs. Si-T2, and SHG44-0 vs. SHG44-T2.

Semi-quantitative reverse transcription-polymerase chainreaction $(R T-P C R)$. Total RNA was extracted after being transfected from the above cells, using TRIzol (Gibco-BRL), according to the manufacturer's instructions. Complementary DNA (cDNA) was generated from total RNA, using M-MLV RT (MBI, Fermentas). To detect ppGalNAc-T2, MMP-2, MMP-9, TGF- $\beta 1$ and TIMP-2 mRNA expression, PCR amplification was performed according to the following thermal cycling parameters: 1 cycle at $94^{\circ} \mathrm{C}$ for $5 \mathrm{~min} ; 32$ cycles at $94^{\circ} \mathrm{C}$ for $45 \mathrm{sec}, 60^{\circ} \mathrm{C}$ for $50 \mathrm{sec}$ and $72^{\circ} \mathrm{C}$ for $1 \mathrm{~min}$; and a final extension cycle at $72^{\circ} \mathrm{C}$ for $10 \mathrm{~min}$. The primer sequences for the genes and expected product sizes were as follows: 5'-AAGAAAGACCTTCATCACAGCAATGGAG AA-3' (forward) and 5'-ATCAAAACCGCCCTTCAAGTCAG CA-3' (reverse) for ppGalNAc-T2 (669 bp); 5'-CATGTAC GTTGCTATCCAGGC-3' (forward) and 5'-CTCCTTAATG TCACGCACGAT-3' (reverse) for $\beta$-actin (250 bp). The annealing temperature of ppGalNAc-T2 was $60^{\circ} \mathrm{C}$, MMP-2, MMP-9, TGF- $\beta 1$ and TIMP-2 were $55^{\circ} \mathrm{C}$ and $\beta$-actin was $56^{\circ} \mathrm{C}$. The PCR products were analyzed on a $1.5 \%$ agarose gel and their images were captured using a gel documentation system.

Western blot analysis. Western blot analysis was conducted using the standard methods. Rabbit anti-human ppGalNAc$\mathrm{T} 2$ polyclonal antibody ( $\mathrm{pAb}$ ) was purified in our laboratory. Protein from SHG44, SHG44-0 and SHG44-T2 cells was extracted with TRIzol (Invitrogen). Proteins from total cell lysates were separated by $10 \%$ SDS-PAGE gel, transferred to a polyvinylidene difluoride membrane, blocked in 5\% non-fat milk in PBS/Tween-20, and blotted with the anti-human ppGalNAc-T2 pAb and then blotted with goat anti-rabbit IgG. $\beta$-actin was used as the loading control.

Wound healing assay. U251, PE, PE-T2, Si-T2 and Scr-T2 cells were seeded into 6 -well plates at equal numbers $\left(1 \times 10^{5}\right)$ and cultured in medium with $10 \%$ FBS. When the cell confluence reached $\sim 90 \%$, cells were cultured in medium with $10 \%$ FBS and mitomycin $\mathrm{C}(200 \mu \mathrm{g} / \mathrm{ml})$. After $1 \mathrm{~h}$, three artificial 


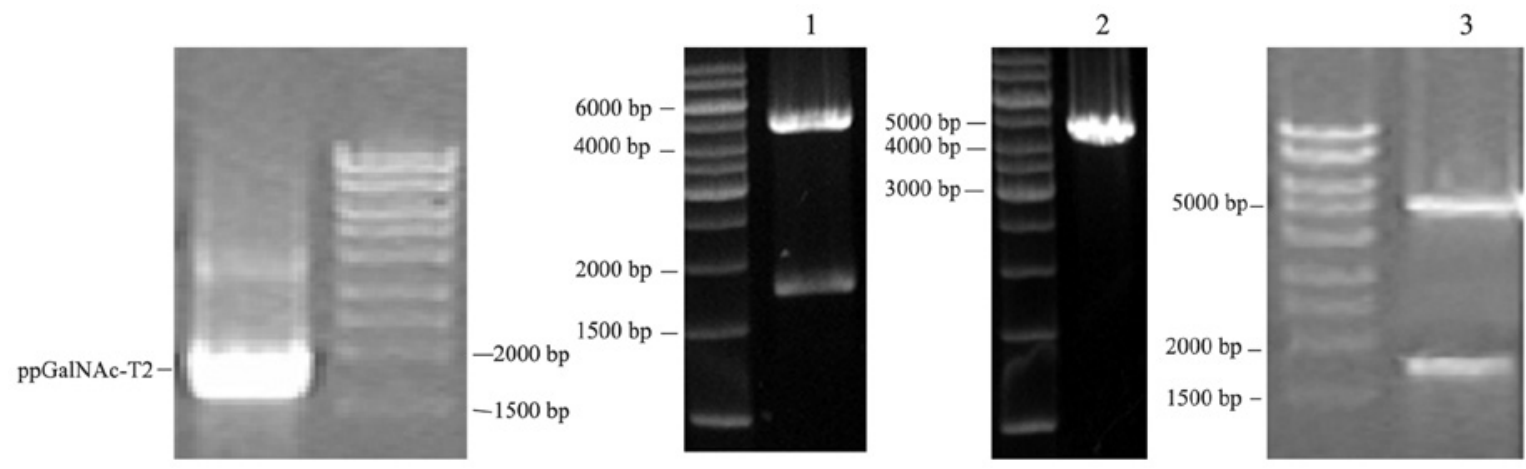

Figure 1. Electrophoretic image. 1, pEGFP-C1-ppGalNAc-T2 plasmid digested with EcoRI and BamHI; 2, pSilenCircle-ppGalNAc-T2Si plasmid digested with StuI; 3, pcDNA3.1-ppGalNAc-T2 plasmid digested with EcoRI and BamHI.

A

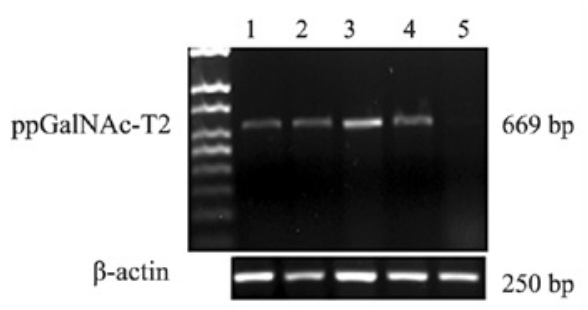

C
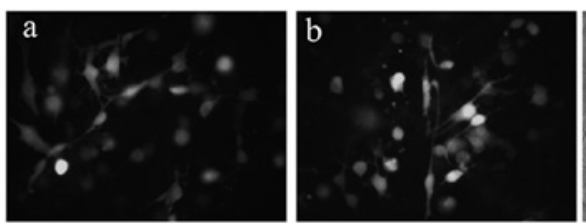
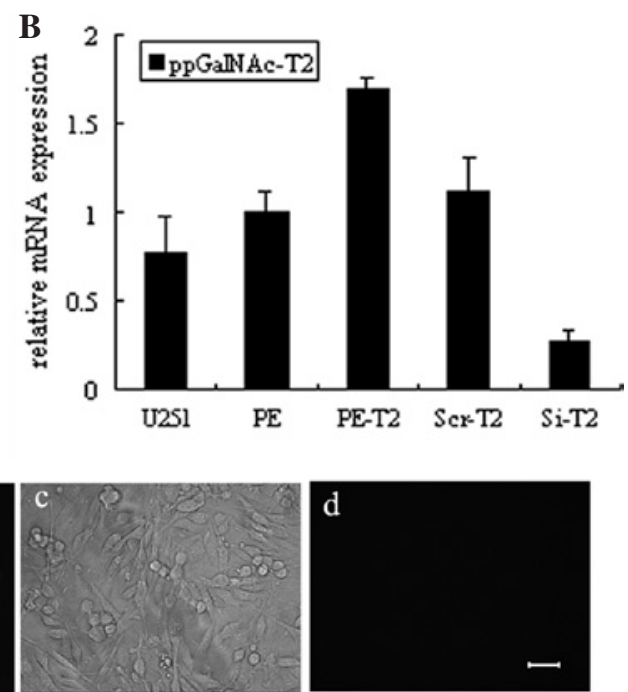

Figure 2. Expression of ppGalNAc-T2 mRNA in the different groups of transfected cells. (A) ppGalNAc-T2 mRNA expression levels were quantified using RT-PCR in the U251 cells. 1, U251; 2, PE; 3, PE-T2; 4, Scr-T2; 5, Si-T2. (B) Densitometric measurements of the bands in the U251 cells. PE-T2 group vs. PE group, Scr-T2 group vs. Si-T2 group $(\mathrm{P}<0.05)$. There was no significant difference between the non-transfected and negative control transfected cells. $(\mathrm{C})$ Expression of GFP fluorescence protein in U251 cells was observed with fluorescence microscopy. Images at $48 \mathrm{~h}$ after transfection. (a) U251 cells transfected with the pEGFP-C1-T2S vector; (b) U251 cells transfected with the pEGFP-C1 vector; (c) transfected cells were observed with light microscopy, (d) nontransfected cells. Bar, $50 \mu \mathrm{m}$.

homogenous wounds were created in the monolayer with a sterile plastic $100-\mu 1$ micropipette tip. Next, the debris was removed by washing the cells with PBS. Cells that migrated into the wounded area or cells with extended protrusion at the border of the wound were visualized and photographed $(=8$ images per treatment) under a light microscope (x200) (21).

Statistical analysis. All experiments were repeated at least three times. The results were presented as means \pm SD. Intergroup comparisons were assessed by ANOVA using SPSS statistical software. $\mathrm{P}<0.05$ was regarded as statistically significant.

\section{Results}

Identification of pcDNA3.1-ppGalNAc-T2, pEGFP-C1-ppGalNAc-T2 and pSilenCircle-T2Si plasmids. The result of restriction enzyme digestion analysis showed the effectiveness of the plasmids (Fig. 1).
Establishment of ppGalNAc-T2-overexpressing or -knockdown cell lines. To investigate the function of ppGalNAc-T2 in glioma cells in vitro, we constructed ppGalNAc-T2-overexpressing or -knockdown cell lines. The ppGalNAc-T2 mRNA levels in the U251 cells were measured by RT-PCR. The ppGalNAc-T2 mRNA expression was increased in the PE-T2 group compared with that in the PE groups and was suppressed in Si-T2 group when compared to the the Scr-T2 group $(\mathrm{P}<0.05)$, while no difference was found between the negative control group and the untreated U251 cells (P>0.05; Fig. 2A and B). Furthermore, the pEGFP-C1 vector is a eukaryotic expression vector including green fluorescent protein (GFP), and the ppGalNAc-T2 protein with GFP in U251 cells transfected with pEGFP-C1-T2S/pEGFP-C1 vectors was observed with fluorescence microscopy (Fig. 2C). Then, the ppGalNAc-T2 mRNA and protein levels in the SHG44 cells were measured by RT-PCR and Western blot analysis, respectively. When compared with their respective control groups, ppGalNAc-T2 transcripts were 


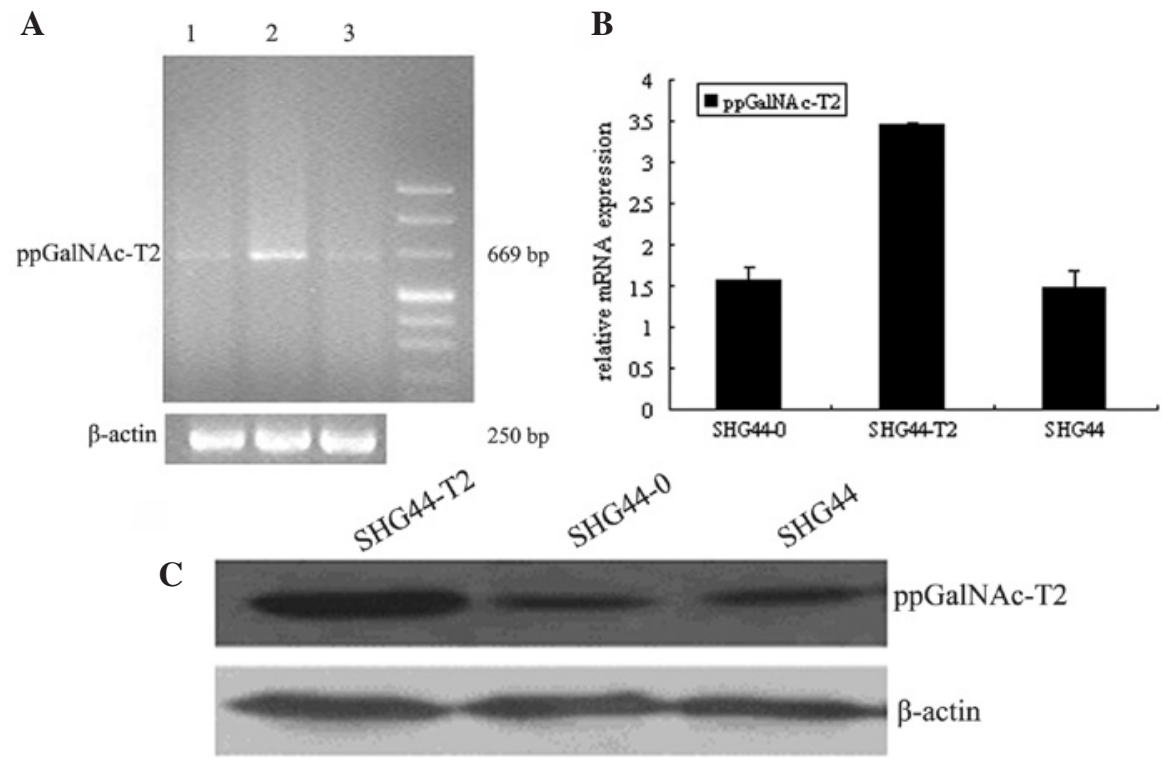

Figure 3. Expression of ppGalNAc-T2 mRNA in the different groups of transfected cells. (A) ppGalNAc-T2 mRNA expression levels quantified using RT-PCR in SHG44 cells. (B) Densitometric measurements of the bands in the the SHG44 cells. SHG44-T2 group vs. SHG44-0 group (P<0.05). There was no significant difference between the non-transfected and negative control transfected cells. (C) Western blotting detection of stable cell lines in the different SHG44 groups. 1, SHG44-0; 2, SHG44-T2; 3, SHG44.

A

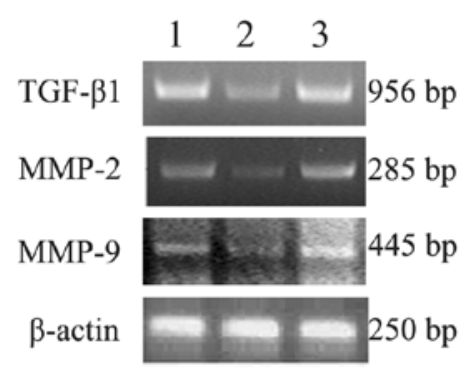

$\mathbf{B}$

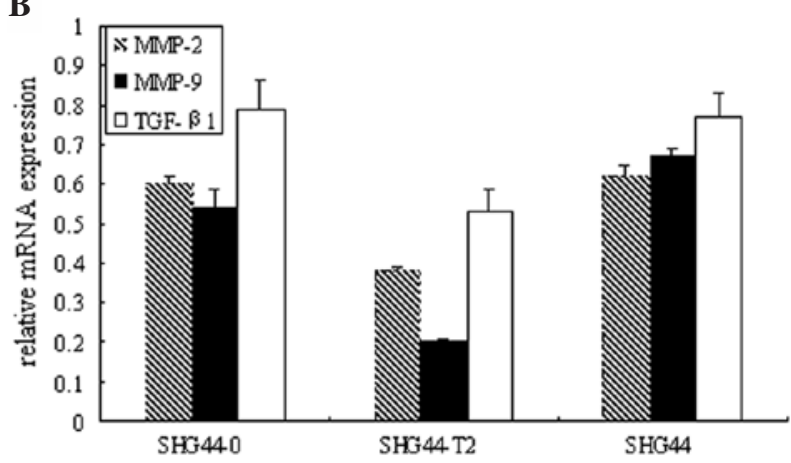

Figure 4. Effect of ppGalNAc-T2 on the expression of MMP-2, MMP-9 and TGF- $\beta 1$ at the mRNA level in the SHG44 cells. (A) The mRNA expression levels of MMP-2, MMP-9 and TGF- $\beta 1$ were determined by semi-quantitative RT-PCR. (B) The quantitative data are shown above. SHG44-T2 group vs. SHG44-0 group $(\mathrm{P}<0.05)$. 1, SHG44; 2, SHG44-T2; 3, SHG44-0.

increased in the SHG44-T2 cells ( $\mathrm{P}<0.05$; Fig. 3A and B). Similar to the RT-PCR results, the expression of ppGalNAc$\mathrm{T} 2$ protein was obviously increased in the SHG44-T2 cells, while no difference was found between the negative control group and untreated cells (Fig. 3C). The above results indicate the successful construction of the ppGalNAcT2-overexpressing or -knockdown cell lines. These stable cell lines can be effectively was further examine the role of ppGalNAc-T2.

Effect of ppGalNAc-T2 on the expression of MMP-2, MMP-9 and TGF- $\beta$ in the different $S H G 44$ cells. We examined whether ppGalNAc-T2 affects the expression of MMP-2, MMP-9 and TGF- $\beta 1$ in SHG44 cells transfected with pcDNA3.1ppGalNAc-T2. Using RT-PCR, we found that the expression of MMP-2, MMP-9 and TGF- $\beta 1$ at the mRNA level was lower in the SHG44-T2 group than that in the SHG44-0 group $(\mathrm{P}<0.05)$, and there was no difference between SHG44-0 and SHG44 cells ( $\mathrm{P}>0.05$; Fig. 4).
Effect of ppGalNAc-T2 on the expression of MMP-2 and TIMP-2 in the different U251 cells. We further examined whether ppGalNAc-T2 affects the expression of MMP-2 and TIMP-2 in U251 cells transfected with the pEGFPC1-ppGalNAc-T2 and pSilenCircle-T2Si plasmids. Using RT-PCR, we found that the expression of MMP-2 at the mRNA level was increased in the ppGalNAc-T2-knockdown cells, but decreased in the ppGalNAc-T2-overexpressing cells. TIMP-2, an endogenous inhibitor of MMP-2, was often found complexed with MMP-2. We found that the expression of TIMP-2 at the mRNA level was contrary to that of MMP-2 $(\mathrm{P}<0.05$; Fig. 5A and $\mathrm{B})$.

ppGalNAc-T2 contributes to glioma cell migration in vitro. To determine whether ppGalNAc-T2 affects glioma cell migration, a wound healing assay was performed to investigate the effect of ppGalNAc-T2 on the different U251 cells. Using this approach, cell migration was found to be significantly enhanced in the knockdown cells $(108.43 \pm 5.86 \mu \mathrm{m})$ 

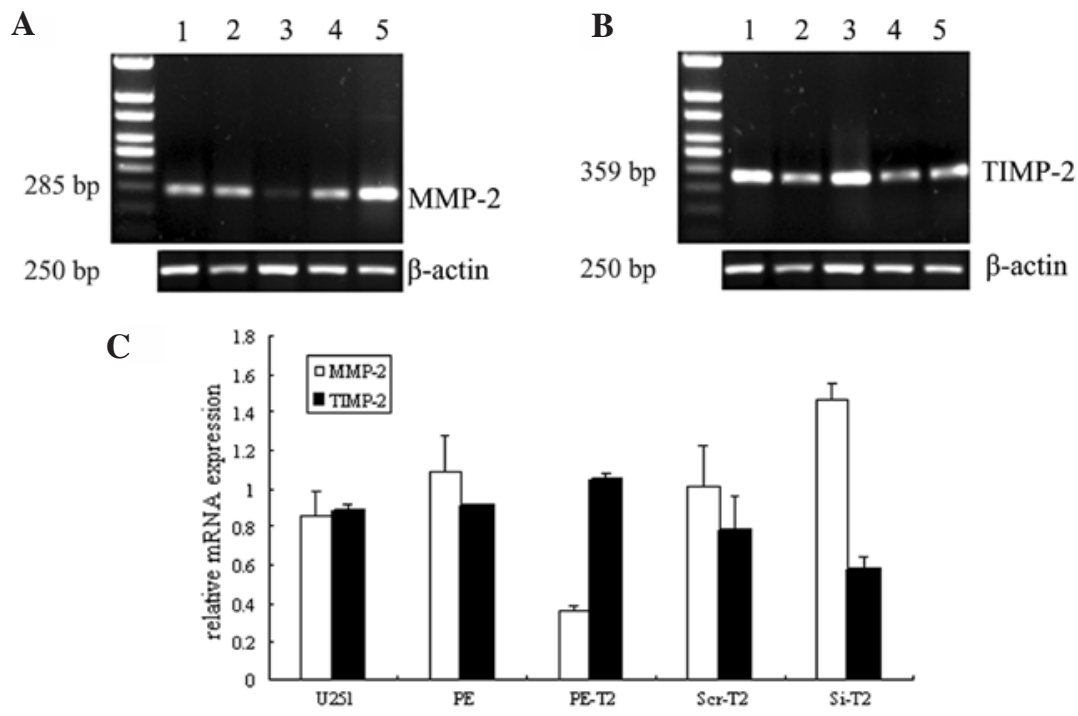

Figure 5. Effect of ppGalNAc-T2 on the expression of MMP-2 and TIMP-2 at the mRNA level in U251 cells. The mRNA expression levels of MMP-2 (A) and TIMP-2 (B) were determined by semi-quantitative RT-PCR. (C) The quantitative data are shown above. PE-T2 group vs. PE group, Si-T2 group vs. Scr-T2 group (P<0.05). 1, U251; 2, PE; 3, PE-T2; 4, Scr-T2; 5, Si-T2.

$\mathbf{A}$
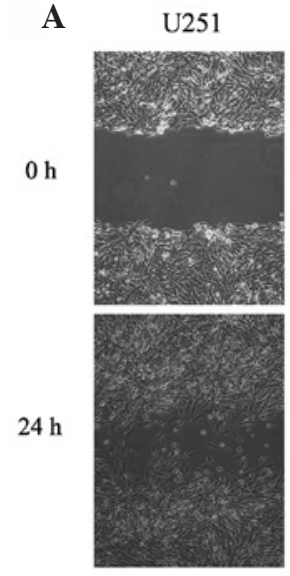

PE
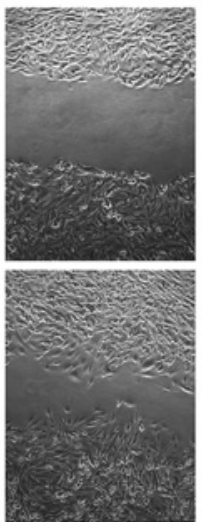

PE-T2
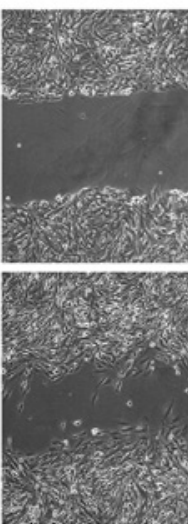

Scr-T2
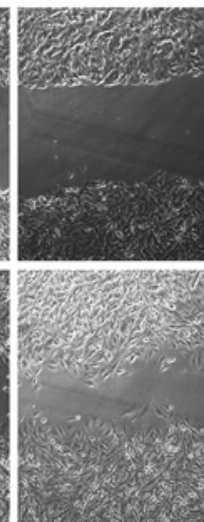

Si-T2
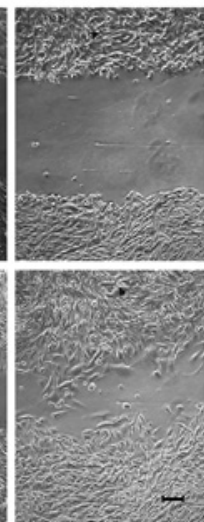

B

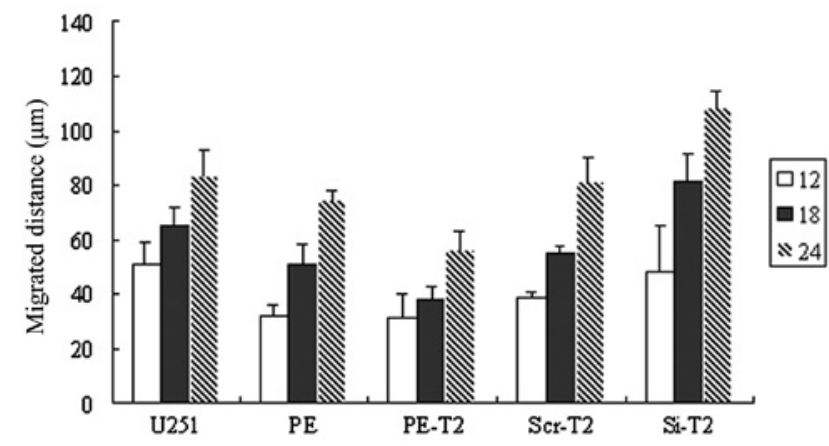

Figure 6. ppGalNAc-T2 inhibits cell metastasis in vitro. (A) Effect of ppGalNAc-T2 on U251 cell migration as detected by the wound healing assay. Cells were observed under a microscope. (B) The distance of the migrated cells in each group ( $\mathrm{n}=3$ ). Results are presented as the mean \pm SD. PE-T2 group vs. PE group, Si-T2 group vs. Scr-T2 group $(\mathrm{P}<0.05)$. Bar, $25 \mu \mathrm{m}$.

and inhibited in the overexpressing cells $(56.82 \pm 5.77 \mu \mathrm{m})$ at $24 \mathrm{~h}$ compared to the negative control cells in vitro $(\mathrm{P}<0.05$; Fig. 6). The cell migration distances for the U215, PE and Scr-T2 cells $(83.68 \pm 9.45,75.04 \pm 3.06$ and $81.60 \pm 8.65 \mu \mathrm{m}$, respectively) at this time point were not significantly different $(\mathrm{P}>0.05)$.

\section{Discussion}

Despite recent advancements in surgical resection and adjuvant therapies, malignant glioma remains one of the most devastating cancers, histologically characterized by a high degree of migration and invasion, particularly glioblastomas. A 
better understanding of the molecular components responsible for glioma migration and invasion may hopefully lead to the development of new treatment methods. New approaches are therefore critical to advance our ability to treat this devastating disease. Here, we investigated the effect of ppGalNAc-T2 on these characteristics of glioma cells.

All cells of every species studied to date are covered by a dense coating of glycans. An increasing body of evidence indicates that sugar chains in glycoproteins are involved in the regulation of cellular functions including cell-cell communication and signal transduction $(2,3)$. The changes in the sugar chains on glycoproteins of cell membrane are closely associated with cell mutations, oncogene activation, metastasis, and invasion of tumor cells. The structures of O-glycans are often unusual or abnormal in cancer, and greatly contribute to the phenotype and biology of cancer cells. Glycosyltransferases that are involved in the formation of glycans play an important role in the progression and metastasis of tumors (7). Studies of their regulation in cancer may reveal the connection between cancerous transformation and glycosylation which may help to understand and control the abnormal biology of tumor cells. There have been some reports on the effect of glycosyltransferases in glioma development. For example, Jiang and $\mathrm{Gu}$ present evidence that $\beta 1,4 \mathrm{GalT} \mathrm{V}$ functions as a positive growth regulator in glioma. $\beta 1,4 \mathrm{GalT} \mathrm{V}$ regulates the invasion, and growth in vivo and in vitro of glioma cells (22). Yamamoto et al confirmed that the overexpression of GnT-V in gliomas led to an increased invasiveness of these tumor cells in vitro (23). In addition, the knockdown of GalTase $\mathrm{V}$ produced enhanced expression of cell surface integrin $\beta 1$ resulting in increased adhesion to fibronectin and suppression of tumor development and metastatic potential in experimental animals $(24,25)$. Therefore, to investigate the roles of such glycosyltransferases may provide insight into our understanding of tumor biology. Our previous study found that ppGalNAc-T2 played a vital role in tumor genesis and progression. In this regard, we modulated the expression level of ppGalNAc-T2, one member of the family of ppGalNAc-Ts, and found that ppGalNAc-T2 regulated invasion and migration of human glioma cells in vitro.

Glioblastomas are characterized by their capacity to infiltrate and migrate to distant sites. Based on evidence, it appears that a major role of MMPs during invasion is not simply to create a path by the breakdown of barrier ECM, but also to rearrange ECM to suit tumor cell migration (26). Regulation of MMP-2 and MMP-9, the most frequently expressed glioma invasion-mediating factors, has been extensively studied. Wild-Bode et al found that MMP-2/MMP-9 activity correlated with glioma cell migration and invasion (27). MMP-2 and MMP-9 were highly expressed in astrocytomas, their expression levels were correlated with the histological grade of tumors, and these two molecules appeared to have a synergistic effect on basement membrane degradation (28). Furthermore, it is now appreciated that the expression and activation of MMP-2 and MMP-9 can also be induced by glycosyltransferases. Our studies have previously shown that the $\beta-1,3-\mathrm{N}$-acetylglucosaminyltransferase- 8 gene controlles the expression and gelatinolytic activity of MMP-2 and TIMP-2 (29). Lith-O-Asp, a novel sialyltransferase inhibitor, decreased the sialic acid modification of integrin- $\beta 1$ and was found to inhibit the expression of MMP-2 and MMP-9 (30). In N-acetylglucosaminyltransferase-V-overexpressing human colon cancer cells, the aberrantly glycosylated TIMP-1 showed weaker inhibition of both MMP-2 and MMP-9, and this aberrancy was closely associated with cancer cell invasion and metastasis in vivo as well as in vitro (31). In glioma cells, TGF- $\beta 1$ has been implicated in the migration and invasion in vitro (32). In particular, the exogenous growth factors investigated are capable of regulating the secretion of MMP-2 and MMP-9 by human brain tumors in vitro. The most effective stimulators of the MMPs are TGF- $\beta 1$ and TGF- $\beta 2$ (33).

Thus, we examined the effect of ppGalNAc-T2 on expression of MMP-2, MMP-9 and TGF- $\beta 1$ in SHG44 cells. Our study revealed that expression of ppGalNAc-T2 had an inverse correlation with the expression of MMP-2, MMP-9 and TGF- $\beta 1$ at the mRNA level. We also investigated the effect of ppGalNAc-T2 on the expression of MMP-2 and TIMP-2 in U251 cells. TIMP-2 is a secreted protein which can complex with MMP-2 and is involved in regulating the activity and activation of individual MMP-2 (34). It appears that TIMP-2 has a direct correlation with the expression of ppGalNAc-T2, contrary to MMP-2. This result was confirmed by a previous study which found that the balance of MMP-2 and TIMP-2 is correlated with tumor growth and metastasis (35).

Wound healing assay is a simple and inexpensive method to study cell migration in vitro, and is a popular method for studying cell movement. We used this technique to investigate the effect of ppGalNAc-T2 on the migration of U251 cells. Our results showed that when ppGalNAc-T2 was up-regulated, cells migrated slower. Consistent with this, knockdown of ppGalNAcT2 in U251 cells facilitates a more rapid wound healing.

In our study, the expression of MMP-2 was higher in the ppGalNAc-T2-knockdown cells and lower in the ppGalNAcT2-up-regulated cells than their respective controls. The above results suggest a possible link between MMP-2 and ppGalNAc$\mathrm{T} 2$ in glioma. Modulation of the synthesis of specific glioma cell-surface glycoprotein alters invasion and metastasis in a manner that may have significant therapeutic potential.

\section{Acknowledgements}

This study was supported by the National Natural Science Foundation of China to Q.C. (no. 30670462).

\section{References}

1. Ohtsubo K and Marth JD: Glycosylation in cellular mechanisms of health and disease. Cell 126: 855-867, 2006.

2. Saxon E and Bertozzi CR: Chemical and biological strategies for engineering cell surface glycosylation. Annu Rev Cell Dev Biol 17: 1-23, 2001.

3. Dwek RA: Glycobiology: 'towards understanding the function of sugars'. Biochem Soc Trans 23: 1-25, 1995.

4. Li M, Song L and Qin X: Glycan changes: cancer metastasis and anti-cancer vaccines. J Biosci 35: 665-673, 2010.

5. Tarp MA and Clausen H: Mucin-type O-glycosylation and its potential use in drug and vaccine development. Biochim Biophys Acta 1780: 546-563, 2008.

6. Wu YM, Nowack DD, Omenn GS and Haab BB: Mucin glycosylation is altered by pro-inflammatory signaling in pancreatic cancer cells. J Proteome Res 8: 1876-1886, 2009.

7. Brockhausen I: Pathways of O-glycan biosynthesis in cancer cells. Biochim Biophys Acta 1473: 67-95, 1999.

8. Ten Hagen KG, Fritz TA and Tabak LA: All in the family: the UDP-GalNAc:polypeptide $\mathrm{N}$-acetylgalactosaminyltransferases. Glycobiology 13: R1-R16, 2003. 
9. Rajpert-De Meyts E, Poll SN, Goukasian I, et al: Changes in the profile of simple mucin-type O-glycans and polypeptide GalNAc-transferases in human testis and testicular neoplasms are associated with germ cell maturation and tumour differentiation. Virchows Arch 451: 805-814, 2007.

10. Liu Z, Shen L, Xu L, Sun X, Zhou J and Wu S: Down-regulation of $\beta-1,3-\mathrm{N}$-acetylglucosaminyltransferase- 8 by siRNA inhibits the growth of human gastric cancer. Mol Med Rep 4: 497-503, 2011.

11. Qiu H, Guo XH, Mo JH, Jin MF, Wu SL and Chen HL: Expressions of polypeptide: $\mathrm{N}$-acetylgalactosaminyltransferase in leukemia cell lines during 1,25-dihydroxyvitamin D3-induced differentiation. Glycoconj J 23: 575-584, 2006.

12. Demuth T and Berens ME: Molecular mechanisms of glioma cell migration and invasion. J Neurooncol 70: 217-228, 2004.

13. Onishi M, Ichikawa T, Kurozumi K and Date I: Angiogenesis and invasion in glioma. Brain Tumor Pathol 28: 13-24, 2011.

14. Stetler-Stevenson WG, Aznavoorian S and Liotta LA: Tumor cell interactions with the extracellular matrix during invasion and metastasis. Annu Rev Cell Biol 9: 541-573, 1993.

15. Gialeli C, Theocharis AD and Karamanos NK: Roles of matrix metalloproteinases in cancer progression and their pharmacological targeting. FEBS J 278: 16-27, 2011.

16. Forsyth PA, Wong H, Laing TD, et al: Gelatinase-A (MMP-2), gelatinase B (MMP-9) and membrane type matrix metalloproteinase-1 (MT1-MMP) are involved in different aspects of the pathophysiology of malignant gliomas. Br J Cancer 79: 1828-1835, 1999.

17. Rich JN, Shi Q, Hjelmeland M, et al: Bone-related genes expressed in advanced malignancies induce invasion and metastasis in a genetically defined human cancer model. J Biol Chem 278: 15951-15957, 2003.

18. Joshi A and Cao D: TGF-beta signaling, tumor microenvironment and tumor progression: the butterfly effect. Front Biosci 15 : 180-194, 2010

19. Sehgal I and Thompson TC: Novel regulation of type IV collagenase (matrix metalloproteinase-9 and -2) activities by transforming growth factor-betal in human prostate cancer cel lines. Mol Biol Cell 10: 407-416, 1999.

20. Sinpitaksakul SN, Pimkhaokham A, Sanchavanakit N and Pavasant P: TGF- $\beta 1$ induced MMP-9 expression in HNSCC cell lines via Smad/MLCK pathway TGF- $\beta 1$ induced MMP-9 expression in HNSCC cell lines via Smad/MLCK pathway. Biochem Biophys Res Commun 371: 713-718, 2008.

21. Moutasim KA, Nystrom ML and Thomas GJ: Cell migration and invasion assays. Methods Mol Biol 731: 333-343, 2011.

22. Jiang $\mathrm{J}$ and $\mathrm{Gu} \mathrm{J}$ : Beta1,4-galactosyltransferase $\mathrm{V}$ a growth regulator in glioma. Methods Enzymol 479: 3-23, 2010.
23. Yamamoto H, Swoger J, Greene S, et al: $\beta 1,6-\mathrm{N}$-acetylglucosamine-bearing N-glycans in human gliomas: implications for a role in regulating invasivity. Cancer Res 60: 134-142, 2000

24. Johnson FM and Shur BD: The level of cell surface beta-1,4-galactosyltransferase I influences the invasive potential of murine melanoma cells. J Cell Sci 112: 2785-2795, 1999.

25. Jiang J, Chen X, Shen J, et al: Beta1,4-galactosyltransferase V functions as a positive growth regulator in glioma. J Biol Chem 281: 9482-9489, 2006.

26. Nabeshima K, Inoue T, Shimao Y and Sameshima T: Matrix metalloproteinases in tumor invasion: role for cell migration. Pathol Int 52: 255-264, 2002.

27. Wild-Bode C, Weller M and Wick W: Molecular determinants of glioma cell migration and invasion. J Neurosurg 94: 978-984, 2001.

28. Lakka SS, Gondi CS and Rao JS: Proteases and glioma angiogenesis. Brain Pathol 15: 327-341, 2005.

29. Shen L, Liu Z, Tu Y, Xu L, Sun X and Wu S: Regulation of MMP-2 expression and activity by $\beta-1,3-\mathrm{N}$-acetylglucosaminyltransferase- 8 in AGS gastric cancer cells. Mol Biol Rep 8: 1541-1550, 2011.

30. Chen JY, Tang YA, Huang SM, et al: A novel sialyltransferase inhibitor suppresses FAK/paxillin signaling and cancer angiogenesis and metastasis pathways. Cancer Res 71: 473-483, 2011.

31. Kim YS, Hwang SY, Kang HY, et al: Functional proteomics study reveals that $\mathrm{N}$-acetylglucosaminyltransferase $\mathrm{V}$ reinforces the invasive/metastatic potential of colon cancer through aberrant glycosylation on tissue inhibitor of metalloproteinase-1. Mol Cell Proteomics 7: 1-14, 2008.

32. Platten M, Wick W, Wild-Bode C, Aulwurm S, Dichgans J and Weller M: Transforming growth factors beta(1) (TGF-beta(1)) and TGF-beta(2) promote glioma cell migration via up-regulation of alphaV-beta3 integrin expression. Biochem Biophys Res Commun 268: 607-611, 2000.

33. Rooprai HK, Rucklidge GJ, Panou C and Pilkington GJ: The effects of exogenous growth factors on matrix metalloproteinase secretion by human brain tumour cells. Br J Cancer 82: 52-55, 2000.

34. Douglas DA, Shi YE and Sang QA: Computational sequence analysis of the tissue inhibitor of metalloproteinase family. J Protein Chem 16: 237-255, 1997.

35. Blavier L, Henriet $P$, Imren $S$ and Declerck YA: Tissue inhibitors of matrix metalloproteinases in cancer. Ann NY Acad Sci 878: 108-119, 1999. 